\title{
A Highly Sensitive Intensity-Modulated Optical Fiber Magnetic Field Sensor Based on the Magnetic Fluid and Multimode Interference
}

\author{
Yi Huang, Tingyun Wang, Chuanlu Deng, Xiaobei Zhang, Fufei Pang, Xuekun Bai, \\ Weilong Dong, Liangjiang Wang, and Zhenyi Chen
}

Key Laboratory of Specialty Fiber Optics and Optical Access Networks, School of Communication and Information Engineering, Shanghai University, 149 Yanchang Road, Shanghai 200072, China

Correspondence should be addressed to Tingyun Wang; tywang@shu.edu.cn

Received 5 July 2017; Accepted 27 August 2017; Published 9 October 2017

Academic Editor: Zhou Zhi

Copyright (C) 2017 Yi Huang et al. This is an open access article distributed under the Creative Commons Attribution License, which permits unrestricted use, distribution, and reproduction in any medium, provided the original work is properly cited.

\begin{abstract}
Fiber-optic magnetic field sensing is an important method of magnetic field monitoring, which is essential for the safety of civil infrastructures, especially for power plant. We theoretically and experimentally demonstrated an optical fiber magnetic field sensor based on a single-mode-multimode-single-mode (SMS) structure immersed into the magnetic fluid (MF). The length of multimode section fiber is determined based on the self-image effect through the simulation. Due to variation characteristics of the refractive index and absorption coefficient of MF under different magnetic fields, an effective method to improve the sensitivity of SMS fiber structure is realized based on the intensity modulation method. This sensor shows a high sensitivity up to $0.097 \mathrm{~dB} / \mathrm{Oe}$ and a high modulation depth up to $78 \%$ in a relatively linear range, for the no-core fiber (NCF) with the diameter of $125 \mu \mathrm{m}$ and length of $59.8 \mathrm{~mm}$ as the multimode section. This optical fiber sensor possesses advantages of low cost, ease of fabrication, high sensitivity, simple structure, and compact size, with great potential applications in measuring the magnetic field.
\end{abstract}

\section{Introduction}

Reliable and highly sensitive magnetic field sensors are quite important for monitoring the variation of the magnetic field strength of civil infrastructure such as power plant. It is also well known that fiber-optic magnetic field sensor has the advantages of good insulation and strong antielectromagnetic interference. The multimode interference (MMI) in the single-mode-multimode-single-mode (SMS) structure is sensitive to external parameters, such as the refractive index, temperature, and axial strain. Various optical fiber devices using the SMS fiber structure have been proposed and demonstrated, such as temperature sensors [1], refractometers [2-4], bandpass filters [5, 6], wavelength tunable fiber laser [7], and chemical gas detector [8]. For the excellent SMS structure, all the above optical fiber devices have advantages such as the low cost, ease of fabrication, and compatibility with standard optical fiber devices. However this SMS structure is intrinsically immune to magnetic fields due to the low magnetooptical coefficient of silica. Therefore, it is difficult to achieve the magnetic field sensing if only using a SMS structure. Magnetic fluid (MF), as a stable colloidal suspension of ferromagnetic nanoparticles $(\sim 10 \mathrm{~nm})$ in certain suitable liquid carriers, owns characteristics such as the special magnetooptical property in the form of Faraday effect, changeable refractive index, birefringence, and anisotropy. And the magnetooptical performance can be tuned by applying the external magnetic field [9-11]. Therefore, fiber magnetooptical sensors combined structures through the MF and SMS have been successfully realized [12-14]. The previous wavelength-based demodulation fiber sensing systems are complicated, expensive, bulky, and timeconsuming, which are not suitable for the outdoor real-time monitoring. However, the intensity demodulation method can directly detect the light intensity using a simple photoelectric conversion device and hence own the features of low cost, small size, and fast response time.

In this paper, a magnetic field sensor based on the single-mode-multimode-single-mode (SMS) structure with no-core fiber as the multimode section immersing in MF is 


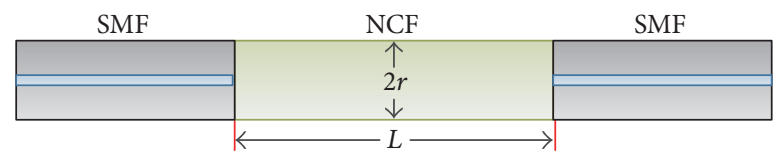

FIGURE 1: Diagram of the SNS fiber structure.

investigated theoretically and experimentally. The proposed magnetic field sensor has the features of intensity encoding and intensity multiplexing capabilities. More importantly, the magnetic field sensor has a simple structure with the potential for high sensitivity intensity-modulated magnetic field measurements. Compared with fiber magnetic sensors based on enhanced Bragg grating [15] and other optical fiber sensors based on MF, such as MF infiltrated photonic crystal fibers [16, 17], Sagnac interferometers [18], cascaded blazing gratings [19], and fiber tapers [20], the proposed scheme features low cost, easy fabrication, and high sensitivity. The proposed structure has higher sensitivity of $0.097 \mathrm{~dB} / \mathrm{Oe}$ and modulation depth of $78 \%$ compared with the existing magnetic field sensors based on SMS and MF, which are mostly wavelength demodulation, such as the magnetic field sensor with sensing sensitivity of $0.01939 \mathrm{~dB} / \mathrm{Oe}$ by using a square no-core fiber [12]. In addition, the proposed structure reduces the complexity of sensing demodulation, which can be realized easily without other auxiliary processes and the splice loss is smaller. Therefore, the intensity demodulation fiber-optic sensors are more practical and applicable than wavelength demodulation sensors. This structure sensor can be used for magnetic field monitoring of civil infrastructure, such as power plant.

\section{Principle and Simulation}

2.1. Principle of SMS. The proposed magnetic field sensor is based on the SMS structure and MF. The SMS fiber structure is fabricated by fusion splicing a specific length section of step-index multimode fiber (MMF) between two sections of standard single-mode fiber (SMF). In this paper, the multimode fiber of the SMS structure is chosen as a section of NCF. The schematic configuration of the proposed SMFNCF-SMF (SNS) fiber structure is shown in Figure 1.

When light transfers from SMF to NCF, a series of linearly polarized modes $\left\{\mathrm{LP}_{n m}\right\}$ of NCF are excited. Due to the circular symmetry of the SMF fundamental mode and the ideal fiber, only the symmetric mode $\left\{\mathrm{LP}_{0 m}\right\}$ can be excited. Hence, the input fundamental mode field distribution of the NCF $E(r, 0)$ can be written in terms of $\left\{\mathrm{LP}_{0 m}\right\}$ modes as follows:

$$
E(r, 0)=\sum_{m=1}^{M} c_{m} \psi_{m}(r)
$$

where $\psi_{m}(r)$ is the field distribution of $\left\{\mathrm{LP}_{0 m}\right\}, r$ is the radial coordinate in the cross section of fiber, $M$ is the number of $\left\{\mathrm{LP}_{0 m}\right\}$, and $c_{m}$ is the excitation coefficient of the $\left\{\mathrm{LP}_{0 m}\right\}$ mode. $c_{m}$ can be calculated by overlap integral between $E(r, 0)$ and $\psi_{m}(r)$, which can be expressed as

$$
c_{m}=\frac{\int_{0}^{\infty} E(r, 0) \psi_{m}(r) r d r}{\int_{0}^{\infty} \psi_{m}(r) \psi_{m}(r) r d r} .
$$

In the NCF section, the field distribution is the result of interferences of all the excited modes $\left\{\mathrm{LP}_{0 m}\right\}$ with different propagation constants, which depends on the length of NCF. Given the length of NCF as $L$, the field distribution $E(r, L)$ can be written as follows:

$$
E(r, z)=\sum_{m=1}^{M} c_{m} \psi_{m}(r) \exp \left(i \beta_{m} L\right),
$$

where $\beta_{m}$ is the propagation constant of the $\left\{\mathrm{LP}_{0 m}\right\}$ mode. When the NCF length meets the relation of $\beta_{m} L=k \pi(k=$ $1,2,3, \ldots)$, this is defined as the reimaging point.

When the output filed distribution $E(r, L)$ from NCF transfers to the second SMF, the power coupling efficiency can be obtained through the overlap integral between $E(r, L)$ and $E(r, 0)$. The normalized output power spectrum can be calculated by

$$
T=10 \log _{10}\left[\frac{\left|\int_{0}^{\infty} E(r, L) E(r, 0) r d r\right|^{2}}{\int_{0}^{\infty}|E(r, L)|^{2} r d r \int_{0}^{\infty}|E(r, 0)|^{2} r d r}\right] .
$$

By simplification, the normalized output power spectrum also can be expressed as

$$
T=10 \log _{10}\left[\frac{\left|\sum_{m=1}^{M} c_{m}{ }^{2} \cdot \exp \left(-i \beta_{m} L\right)\right|^{2}}{\sum_{m=1}^{M} c_{m}{ }^{2}}\right] .
$$

2.2. Simulation of the Magnetic Field Sensing. In the simulation, the SNS structure immersed into MF is designed as the magnetic field sensor, and the MF can be recognized as the cladding of NCF. The SMF has a core diameter of $9 \mu \mathrm{m}$ and the refractive indices of core and cladding are 1.4504 and 1.4447, respectively. The NCF diameter is $125 \mu \mathrm{m}$ and the refractive index is 1.4447. The refractive index of MF (EMG 705 ) is estimated to be about 1.42 [21].

Figure 2 shows the excitation coefficients for $\left\{\mathrm{LP}_{0 m}\right\}$ modes in NCF when the wavelength $\lambda$ is $1550 \mathrm{~nm}$. The mode excitation coefficient first increases and then decreases as mode order increases. When the mode order increases to a certain value of 18 , the mode excitation coefficient is approximately 0 . Moreover, the excitation coefficient distribution characteristic is different for the certain mode order under different wavelength. It means that the interference pattern of all the excited modes also is different.

For the SNS structure, the optimal length of NCF can be determined through the light propagation along NCF, as shown in Figure 3. The reimaging point $R$ within the NCF is at a $z$ position $60 \mathrm{~mm}$. The insets display the enlarged details for the reimaging point $R$ and the coupling point $O$ between SMF and NCF, which indicates that the interference pattern for the two points almost is the same. 


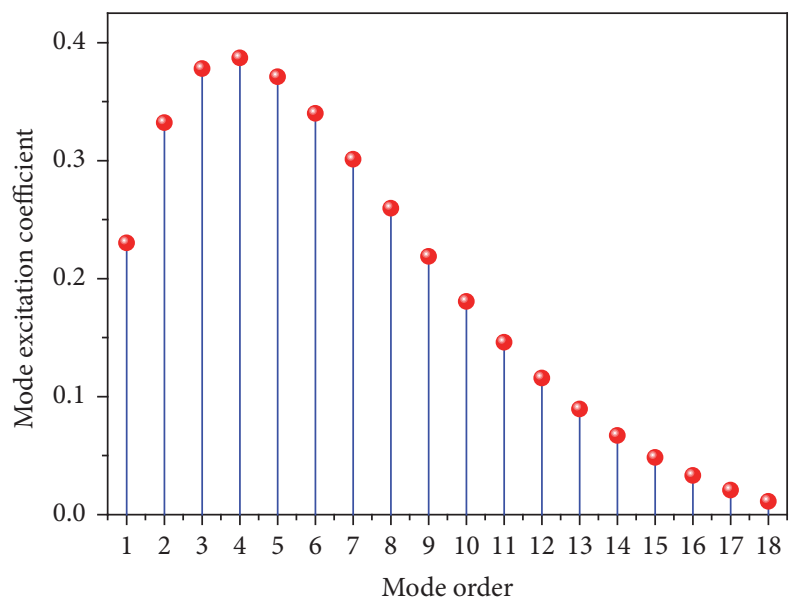

FIGURE 2: Excitation coefficient for modes with different order in NCF under a surrounding RI of 1.42 .

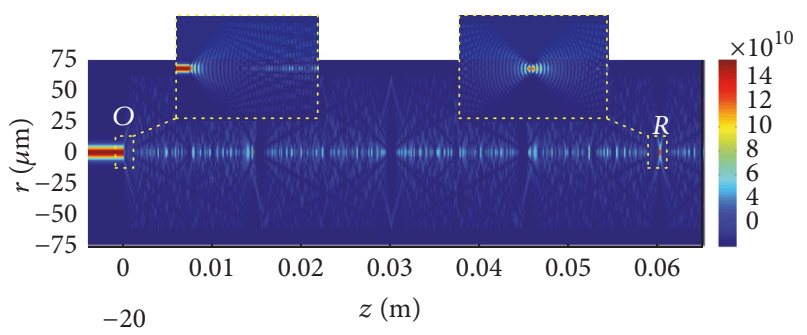

FIGURE 3: Light propagation along the NCF.

The MF is based on a commercial product of EMG 705. Under magnetic field of $0-200 \mathrm{Oe}$, the RI change of MF is approximately 0.003 [22], and the attenuation coefficient $\alpha_{f}$ changes in the order of $10^{4}$ [23]. Due to the great absorption characteristics of MF, (5) can be amended as follows:

$T$

$$
=10 \log _{10}\left[\frac{\left|\sum_{m=1}^{M} c_{m}{ }^{2} \exp \left(-\gamma_{m} L\right) \cdot \exp \left(-i \beta_{m} L\right)\right|^{2}}{\sum_{m=1}^{M} c_{m}{ }^{2}}\right],
$$

where $\gamma_{m}$ is the evanescent absorption coefficient of the $\left\{\mathrm{LP}_{0 m}\right\}$ mode with relation to the refractive index $n_{f}$ and the attenuation coefficient $\alpha_{f}[24,25]$.

Figure 4 shows the calculated transmission spectrum of the SNS structure under different magnetic fields when the NCF length is $59.8 \mathrm{~mm}$. As shown in Figure 4, there are dips (A), (B), and (D) and peak (C) (reimaging point), due to the principle of MMI. The wavelength shifts of dips (A), (B), and (D) are very small, because the RI change of MF under magnetic field varies lightly. However, in the magnetic field sensor based on SMS structure immersing in MF, the wavelength shift is large under different magnetic fields, because the smaller the NCF diameter, the larger the sensitivity of SNS structure [3]. Furthermore, the modulation depths of dips (A), (B), and (D) change greatly, which is derived from the larger change of absorption coefficients

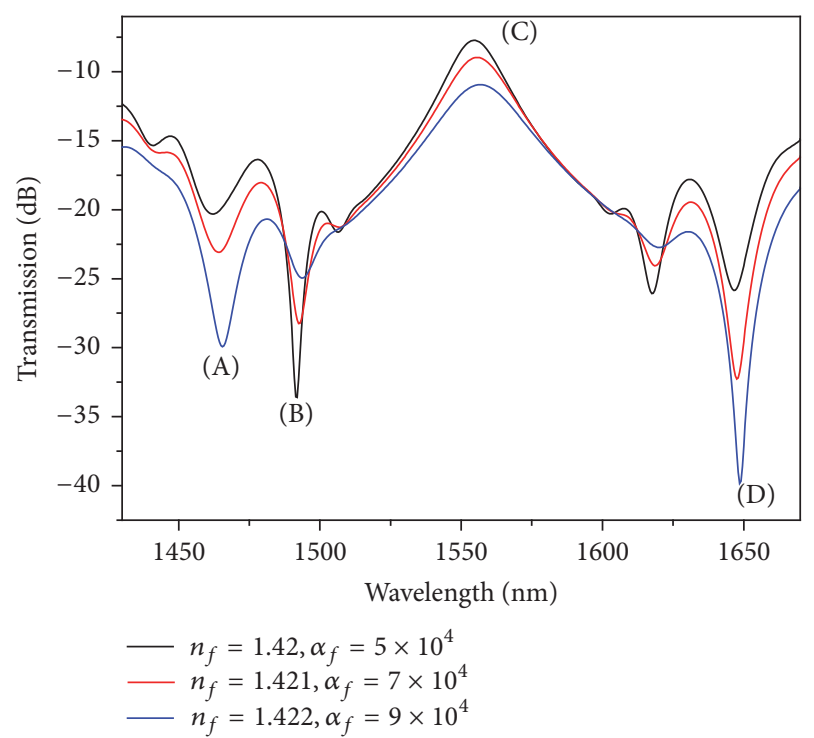

FIGURE 4: The calculated transmission spectra of the SNS structure under different magnetic fields.

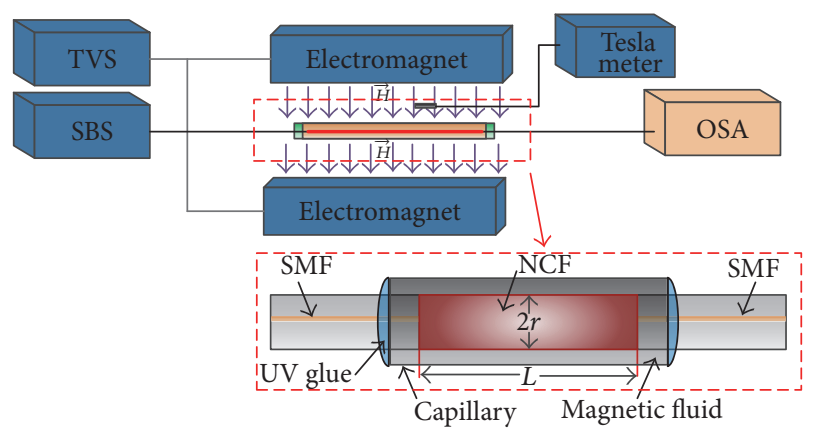

FIgURE 5: Schematic diagram of the experimental setup.

$\gamma_{m}$ of MF under different magnetic fields. Moreover, the reimaging point (peak $(\mathrm{C})$ ) also shows similar changing characteristics. As the attenuation coefficient $\alpha_{f}$ increases with increasing the magnetic field, the demodulation depths of dips (A) and (D) and peak (C) are strengthened; however, the demodulation depth of $\operatorname{dip}(\mathrm{B})$ is weakened.

Therefore, the SNS structure can be designed as a magnetic field sensor based on the intensity modulation. Compared to the previous scheme adopting a combination with the wavelength shift and intensity modulation [12, 13], it can greatly reduce the complexity of sensing demodulation.

\section{Experiments and Results}

The experimental setup of the proposed magnetic field sensor is shown in Figure 5. It consists of a supercontinuum broadband source (SBS) (NKT, SuperK COMPACT), an optical spectrum analyzer (OSA) (Yokogawa AQ6375), a sensor head based on a MMI and the MF, two electromagnets, and a tunable voltage source (TVS). A SBS and an OSA are used to record the transmission spectrum. The sensor 


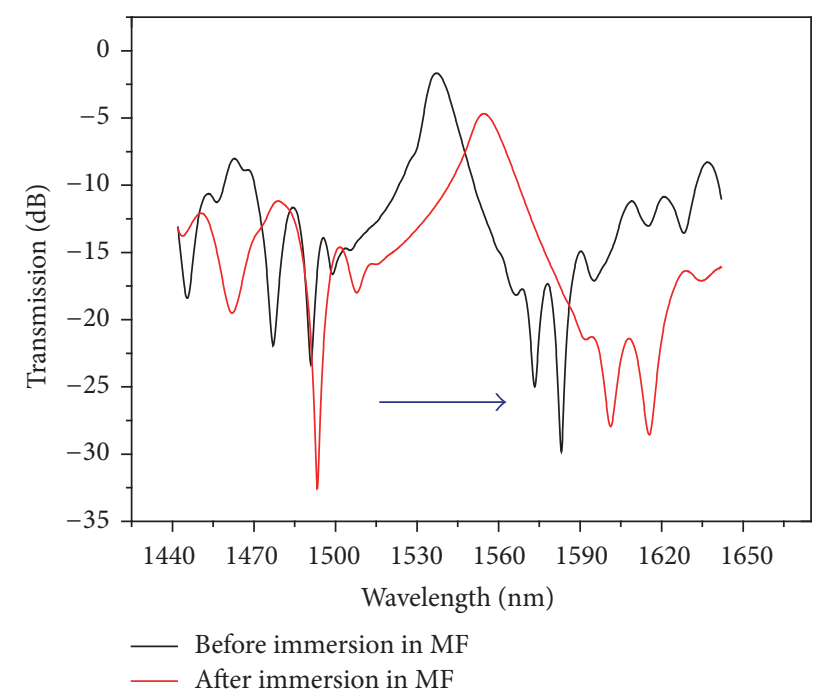

FIgURE 6: The transmission spectra of the proposed sensors with and without the immersion in MF.

head is formed by a MMI immersed in the MF. The MMI is constructed by splicing a section of NCF (Prime Optical Fiber Co. Ltd., NCF125) between two SMFs (SMF-28e, Corning, Inc.). The NCF is made of pure silica, where the radius is $r=62.5 \mu \mathrm{m}$, the length is $L=59.8 \mathrm{~mm}$, and its refractive index is 1.4446. The capillary is infiltrated with MF (EMG705, Ferrotec, Inc.). The density and saturation magnetization of the $\mathrm{MF}$ are $1.19 \mathrm{~g} / \mathrm{cm}^{3}\left(25^{\circ} \mathrm{C}\right)$ and $220 \mathrm{Oe}$, respectively. The two ends of the capillary are sealed with UV glue. The external magnetic field perpendicular to the fiber axis is generated by two electromagnets dynamically tuned by the TVS. The strength of the magnetic field is controlled by tuning the magnitude of the supply current. The magnetic field direction is perpendicular to the optical fiber axis. A Tesla meter (HT 108) with a resolution of $0.1 \mathrm{Oe}$ is used to measure the magnetic field intensity along the perpendicular axis. During the experiment, the ambient temperature is kept at $25^{\circ} \mathrm{C}$.

The transmission spectrum of the MMI before and after immersing into the MF is shown in Figure 6, and a red-shift to long wavelength side occurs when the MMI is immersed into the MF. By immersing the MMI section into the MF and exploiting RI tunability of the MF under varying magnetic field, a magnetic field sensor can be achieved.

Figure 7 shows the transmission characteristics of the proposed sensor by tuning the TVS to change the magnetic field strength $(H)$ ranging from 0 to 240 Oe with a step of $15 \mathrm{Oe}$. Each curve is recorded after $5 \mathrm{~min}$ after specific $H$ is applied to ensure the stabilization of the spectrum. Three distinct interference dips and a peak are observed in the wavelength range of 1450 to $1625 \mathrm{~nm}$; that is, the interference dip around $1461 \mathrm{~nm}$ is referred to as dip (A), while around $1495 \mathrm{~nm}$ it is referred to as dip (B) and around $1617 \mathrm{~nm}$ it is referred to as dip (D), and the interference peak around $1552 \mathrm{~nm}$ is referred to as peak (C). peak (C) is the reimaging point. With the magnetic field increases, the depths of the interference dips (A) and (D) monotonously increase, and it is reversed for dip (B). Moreover, the interference peak

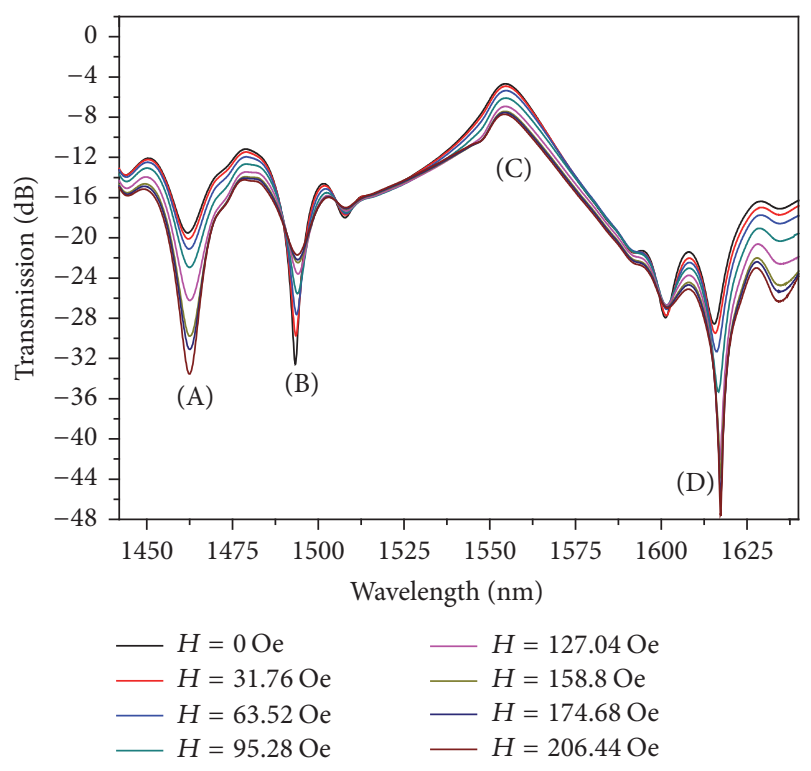

FIGURE 7: Transmission spectra of the proposed sensing structure at magnetic field strength ranging from 0 to 206.44 Oe.

(C) becomes shallower and shallower monotonously. The changing characteristics of experimental spectral response confirm well the simulation results.

The experimental results can be explained by the tunable refractive index $n_{f}$ and evanescent field absorption $\gamma_{m}$ of the MF. When an external magnetic field is applied to the MF, the magnetic particles form the agglomeration and then chainlike clusters. The chain-like cluster of particles changes the extinction coefficient of MF, which determines the attenuation coefficient $\alpha_{f}[23,24]$. The intensity attenuation is mainly caused by the evanescent field absorption coefficient $\gamma_{m}$ of the MF. According to the evanescent field absorption theory in a multimode fiber [24], the change of $\gamma_{m}$ is approximately in the order of $10^{1}$, and its influence on the intensity attenuation is not negligible, whereas because the change of $n_{f}$ is only of the order of $10^{-3}$, the wavelength shift on the transmission spectrum caused by the MMI is almost constant.

Figure 8 is an enlarged view of dip (A) and peak (C). The intensity of the interference dip (A) changes from $-19.77 \mathrm{~dB}$ to $-34.70 \mathrm{~dB}$ as shown in Figure 8(a) and the intensity of the interference peak (C) changes from $-4.79 \mathrm{~dB}$ to $-7.75 \mathrm{~dB}$ as shown in Figure 8(b) with the increase of the magnetic field strength. As in the principle simulation section, on one hand the center wavelength of dip (A) and peak (C) hardly shifts with the change of the magnetic field; on the other hand the intensities of peak (C) decrease and the intensities of dip (A) increase with an increase of the magnetic field because of the tunable RI and absorption coefficient of MF. Similarly, the intensity of dips (B) and (D) changes from $-32.57 \mathrm{~dB}$ to $-21.70 \mathrm{~dB}$ and $-28.56 \mathrm{~dB}$ to $-47.67 \mathrm{~dB}$, respectively.

The intensity of $\operatorname{dip}(\mathrm{A})$ and peak (C) as a function of external magnetic field is shown in Figure 9(a), indicating that the intensity is linearly proportionally to magnetic field strength in the range of 63.52-142.92 Oe and 79.4-206.44 Oe, 


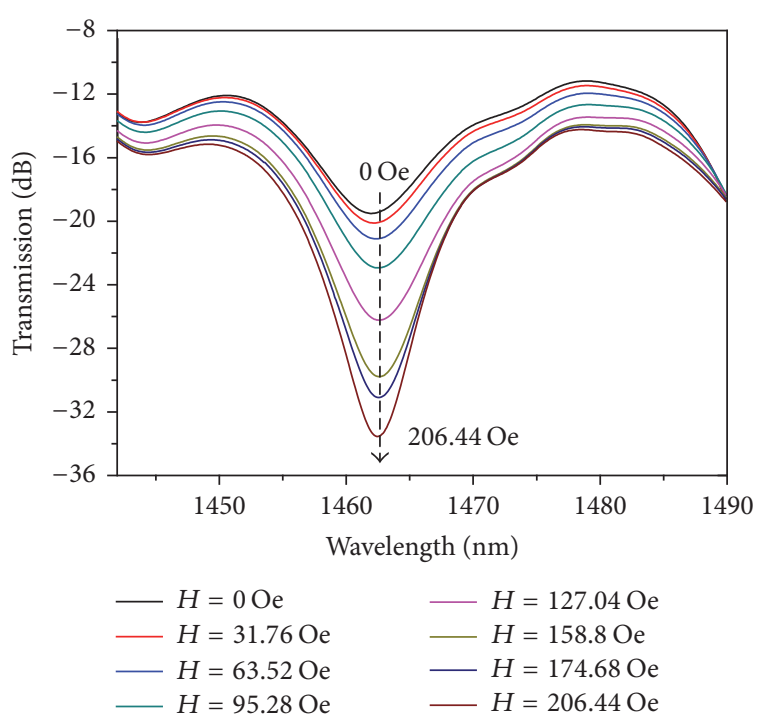

(a)

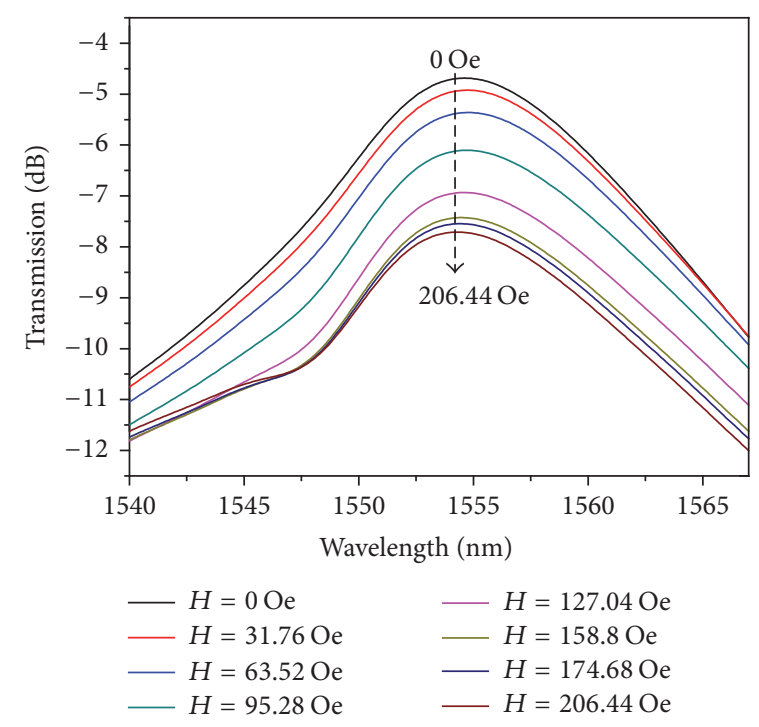

(b)

FIGURE 8: Transmission spectra of (a) dip (A) and (b) peak (C) subjected to different strengths of magnetic field.

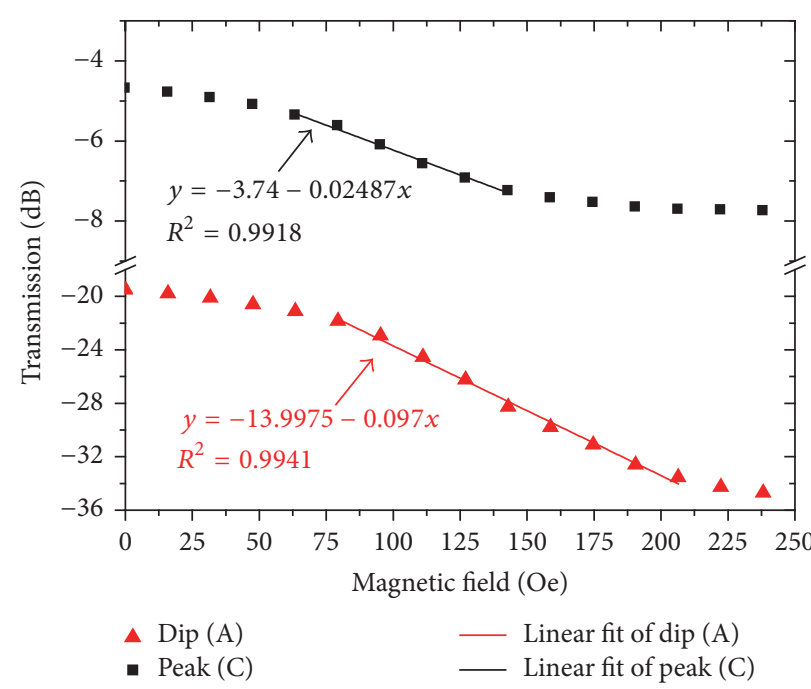

(a)

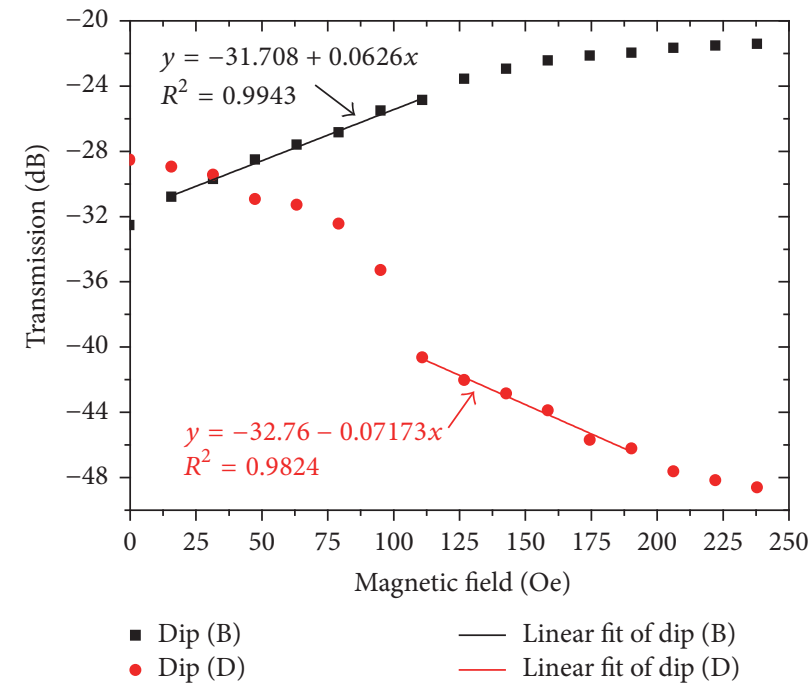

(b)

FIgURE 9: Intensities of (a) dip (A), peak (C) and (b) dip (B), dip (D) as functions of magnetic field.

respectively. Such linear relationship benefits practical sensing application [26]. By linear fitting, the maximum sensitivities of magnetic field are found to be $0.097 \mathrm{~dB} / \mathrm{Oe}$ and $0.0248 \mathrm{~dB} /$ Oe for dip (A) and peak (C), respectively. Similarly, the linearly changing range for dips (B) and (D) is 15.88-111.16 Oe and 111.16-190.56 Oe, respectively, with the maximum sensitivities as $0.0626 \mathrm{~dB} / \mathrm{Oe}$ and $0.071 \mathrm{~dB} / \mathrm{Oe}$, respectively, which is shown in Figure 9(b). It is made clear that the intensity of dips (A), (B), and (D) is larger than that of peak (C), with the sensitivity of dip (A) being the largest one.

Sensitivity is an important parameter of sensor performance. High sensitivity is usually preferable in practical application. Besides, considering the comprehensive sensing properties of the proposed magnetic field sensor, the modulation depth $(M)$ is defined as [26]

$$
M=1-\frac{T_{\min }}{T_{\max }}=\frac{T_{\max }-T_{\min }}{T_{\max }},
$$

where $T_{\max }$ and $T_{\min }$ are the maximum and minimum of the intensity in transmission spectrum with the magnetic field, respectively, and $M$ is the contrast ratio between $T_{\max }$ and $T_{\min }$. The modulation depth as a function is shown in Figure 10. The modulation depth of dip (A), peak (C), dip (B), and dip (D) is $78 \%, 65 \%, 23 \%$, and $61 \%$, respectively, which can be used for the sensing demodulation.

From (6), the transmission of dips (A), (B), and (D) and peak $(\mathrm{C})$ is related to the excitation coefficient $c_{m}$, the 


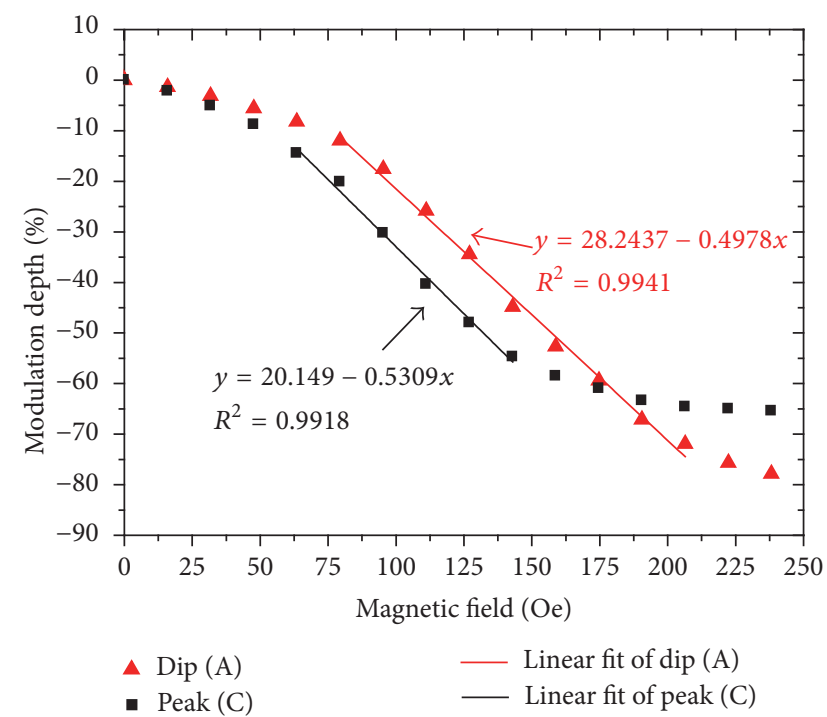

(a)

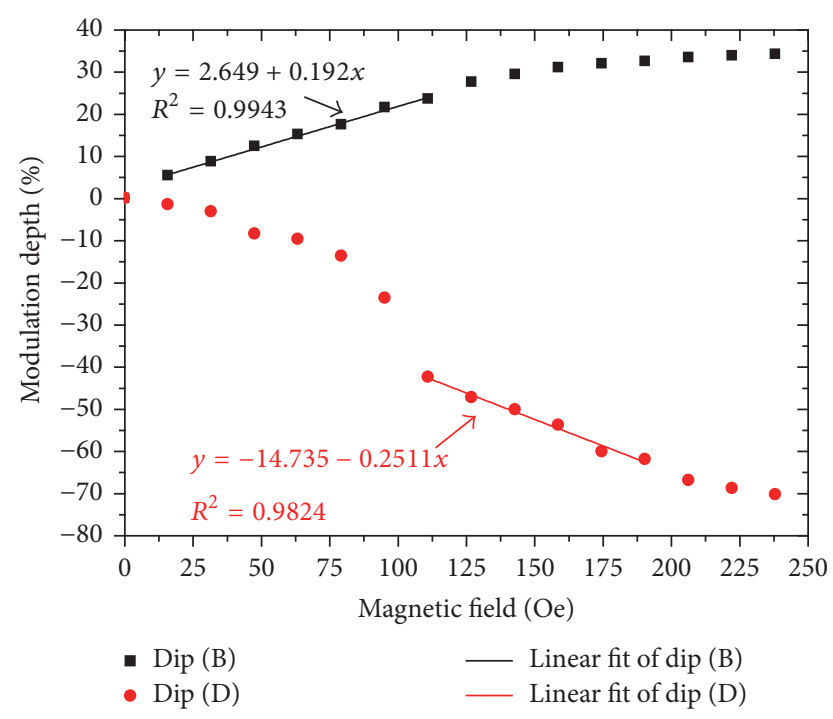

(b)

Figure 10: Modulation depth of (a) dip (A), peak (C) and (b) dip (B), dip (D) versus the strength of magnetic field.

evanescent absorption coefficient $\gamma_{m}$, and the mode phase $\beta_{m} L$. For peak (C), it is a self-image point and the ideal transmission is $100 \%$ if the evanescent absorption coefficient is zero. However, the intensity of peak (C) will vary a little if the evanescent absorption coefficient exists. Similarly, the intensities of dips (A), (B), and (D) will also vary if the evanescent absorption coefficient exists. However, the variations of intensities of dips (A), (B), and (D) will have larger variations because the previous values are on a much lower level, while the intensity of peak (C) is on a larger level.

Considering the sensitivity and modulation depth, dip (A) with a larger sensitivity is more suitable for practical sensing application. The sensitivity of the proposed structure is higher $(0.097 \mathrm{~dB} / \mathrm{Oe})$ than that of [12] $(0.01939 \mathrm{~dB} / \mathrm{Oe})$.

\section{Conclusions}

In conclusion, an intensity-modulated magnetic field sensor based on the combination with SNS structure and MF is investigated theoretically and experimentally. This sensor has a high sensitivity of $0.097 \mathrm{~dB} / \mathrm{Oe}$ and high modulation depth of $78 \%$ in a relatively linear range. It has features including low cost, ease of fabrication, compactness, and high sensitivity, which suggests that the proposed sensor is simple and effective in the measurement of magnetic field.

\section{Conflicts of Interest}

The authors declare no conflicts of interest.

\section{Acknowledgments}

This work is supported by the National Natural Science Foundation of China (NSF) (61475096, 61520106014, 61635006, 61675126, and 61475095).

\section{References}

[1] S. M. Tripathi, A. Kumar, R. K. Varshney, Y. B. P. Kumar, E. Marin, and J. P. Meunier, "Strain and temperature sensing characteristics of single-mode-multimode-single-mode structures," Journal of Lightwave Technology, vol. 27, no. 13, pp. 2348-2356, 2009.

[2] Z. B. Liu, Z. Tan, B. Yin, Y. Bai, and S. Jian, "Refractive index sensing characterization of a singlemode-claddinglesssinglemode fiber structure based fiber ring cavity laser," Optics Express, vol. 22, no. 5, pp. 5037-5042, 2014.

[3] Q. Wu, Y. Semenova, P. Wang, and G. Farrell, "High sensitivity SMS fiber structure based refractometer - Analysis and experiment," Optics Express, vol. 19, no. 9, pp. 7937-7944, 2011.

[4] X. Zhang, H. Shao, Y. Yang, H. Pan, F. Pang, and T. Wang, "Refractometry with a tailored sensitivity based on a singlemode-capillary-single-mode fiber structure," IEEE Photonics Journal, vol. 9, no. 2, pp. 1-8, 2017.

[5] W. S. Mohammed, P. W. E. Smith, and X. Gu, "All-fiber multimode interference bandpass filter," Optics Letters, vol. 31, no. 17, pp. 2547-2549, 2006.

[6] J. E. Antonio-Lopez, A. Castillo-Guzman, D. A. May-Arrioja, R. Selvas-Aguilar, and P. Likamwa, "Tunable multimodeinterference bandpass fiber filter," Optics Letters, vol. 35, no. 3, pp. 324-326, 2010.

[7] A. Castillo-Guzman, J. E. Antonio-Lopez, R. Selvas-Aguilar, D. A. May-Arrioja, J. Estudillo-Ayala, and P. LiKamWa, "Widely tunable erbium-doped fiber laser based on multimode interference effect," Optics Express, vol. 18, no. 2, pp. 591-597, 2010.

[8] X. Lan, J. Huang, Q. Han et al., "Fiber ring laser interrogated zeolite-coated singlemode-multimode- singlemode structure for trace chemical detection," Optics Letters, vol. 37, no. 11, pp. 1998-2000, 2012.

[9] C. Y. Hong, H. E. Horng, F. C. Kuo, S. Y. Yang, H. C. Yang, and J. M. Wu, "Evidence of multiple states of ordered structures and a phase transition in magnetic fluid films under perpendicular magnetic fields," Applied Physics Letters, vol. 75, no. 15, pp. 21962198, 1999. 
[10] S. Y. Yang, J. J. Chieh, H. E. Horng, C.-Y. Hong, and H. C. Yang, "Origin and applications of magnetically tunable refractive index of magnetic fluid films," Applied Physics Letters, vol. 84, no. 25, pp. 5204-5206, 2004.

[11] J. E. Antonio-Lopez, J. J. Sanchez-Mondragon, P. LiKamWa, and D. A. May-Arrioja, "Fiber-optic sensor for liquid level measurement," Optics Letters, vol. 36, no. 17, pp. 3425-3427, 2011.

[12] W. Lin, Y. Miao, H. Zhang, B. Liu, Y. Liu, and B. Song, "Fiberoptic in-line magnetic field sensor based on the magnetic fluid and multimode interference effects," Applied Physics Letters, vol. 103, no. 15, Article ID 151101, 2013.

[13] H. Wang, S. Pu, N. Wang, S. Dong, and J. Huang, "Magnetic field sensing based on singlemode-multimode-singlemode fiber structures using magnetic fluids as cladding," Optics Letters, vol. 38, no. 19, pp. 3765-3768, 2013.

[14] S. Dong, S. Pu, and J. Huang, "Magnetic field sensing based on magneto-volume variation of magnetic fluids investigated by air-gap Fabry-Pérot fiber interferometers," Applied Physics Letters, vol. 103, no. 11, Article ID 111907, 2013.

[15] Y. Dai, M. Yang, G. Xu, and Y. Yuan, "Magnetic field sensor based on fiber Bragg grating with a spiral microgroove ablated by femtosecond laser," Optics Express, vol. 21, no. 14, pp. 1738617391, 2013.

[16] H. V. Thakur, S. M. Nalawade, S. Gupta, R. Kitture, and S. N. Kale, "Photonic crystal fiber injected with $\mathrm{Fe} 3 \mathrm{O} 4$ nanofluid for magnetic field detection," Applied Physics Letters, vol. 99, no. 16, Article ID 161101, 2011.

[17] P. Zu, C. Chiu Chan, T. Gong, Y. Jin, W. Chang Wong, and X. Dong, "Magneto-optical fiber sensor based on bandgap effect of photonic crystal fiber infiltrated with magnetic fluid," Applied Physics Letters, vol. 101, no. 24, Article ID 241118, 2012.

[18] P. Zu, C. C. Chan, W. S. Lew et al., "Magneto-optical fiber sensor based on magnetic fluid," Optics Letters, vol. 37, no. 3, pp. 398400, 2012.

[19] P. Childs, A. Candiani, and S. Pissadakis, "Optical fiber cladding ring magnetic field sensor," IEEE Photonics Technology Letters, vol. 23, no. 13, pp. 929-931, 2011.

[20] S. Pu, X. Chen, Y. Chen et al., "Fiber-optic evanescent field modulator using a magnetic fluid as the cladding," Journal of Applied Physics, vol. 99, no. 9, p. 093516, 2006.

[21] H. E. Horng, C.-Y. Hong, S. Y. Yang, and H. C. Yang, "Designing the refractive indices by using magnetic fluids," Applied Physics Letters, vol. 82, no. 15, pp. 2434-2436, 2003.

[22] Y. F. Chen, S. Y. Yang, W. S. Tse, H. E. Horng, C. Y. Hong, and H. C. Yang, "Thermal effect on the field-dependent refractive index of the magnetic fluid film," Applied Physics Letters, vol. 82, no. 20, pp. 3481-3483, 2003.

[23] X. Fang, Y. Xuan, and Q. Li, "Theoretical investigation of the extinction coefficient of magnetic fluid," Journal of Nanoparticle Research, vol. 15, no. 5, pp. 1-12, 2013.

[24] X. Fang, Y. Xuan, and Q. Li, "Measurement of the extinction coefficients of magnetic fluids," Nanoscale Research Letters, vol. 6, no. 1, pp. 1-5, 2011.

[25] V. Ruddy, B. D. MacCraith, and J. A. Murphy, "Evanescent wave absorption spectroscopy using multimode fibers," Journal of Applied Physics, vol. 67, no. 10, pp. 6070-6074, 1990.

[26] J. Tang, S. Pu, S. Dong, and L. Luo, "Magnetic field sensing based on magnetic-fluid-clad multimode-singlemodemultimode fiber structures," Sensors (Switzerland), vol. 14, no. 10, pp. 19086-19094, 2014. 


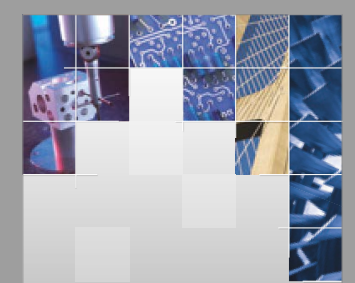

\section{Enfincering}
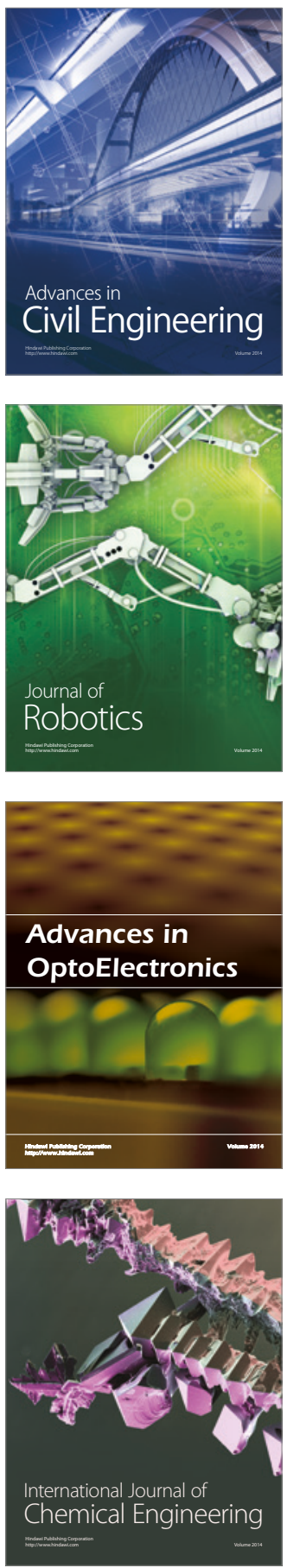

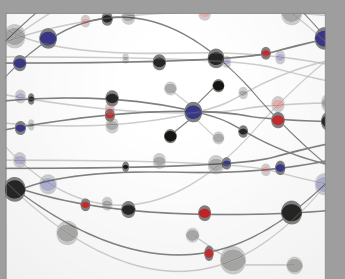

The Scientific World Journal

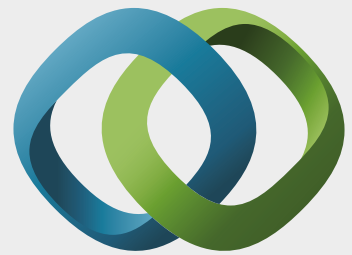

\section{Hindawi}

Submit your manuscripts at

https://www.hindawi.com
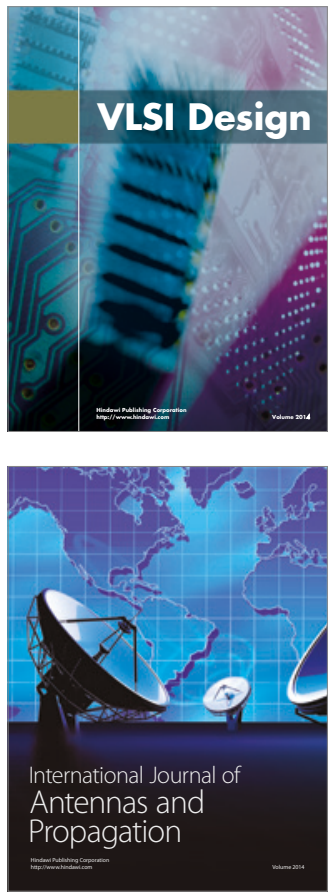

\section{Rotating}

Machinery
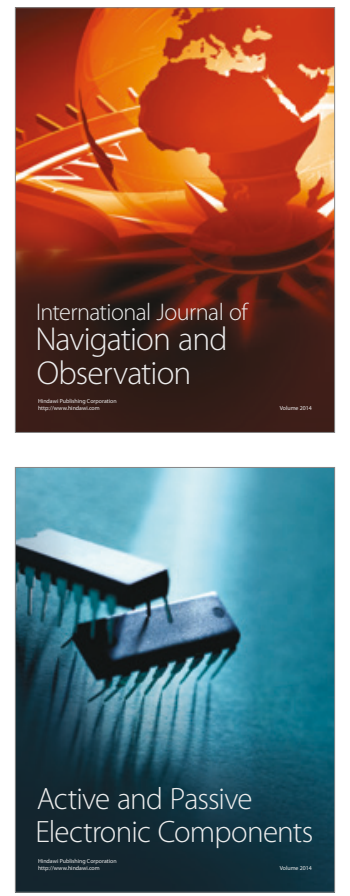
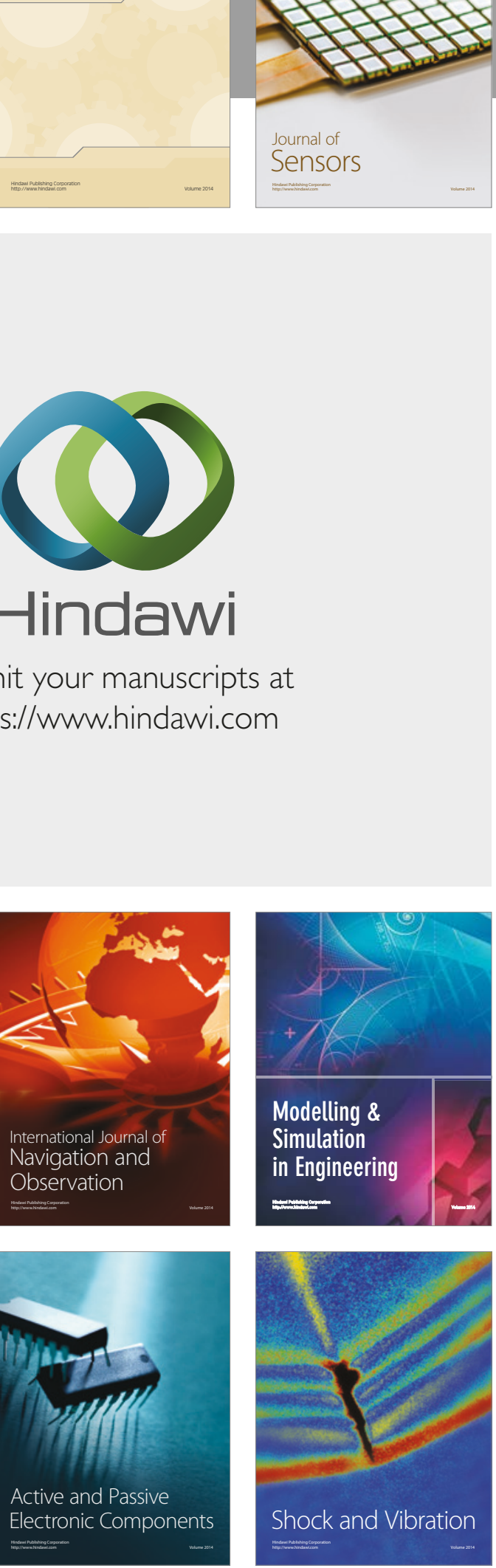
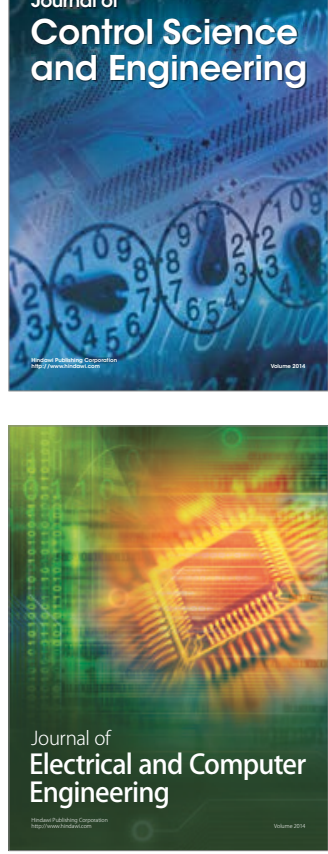

Distributed

Journal of

Control Science

and Engineering
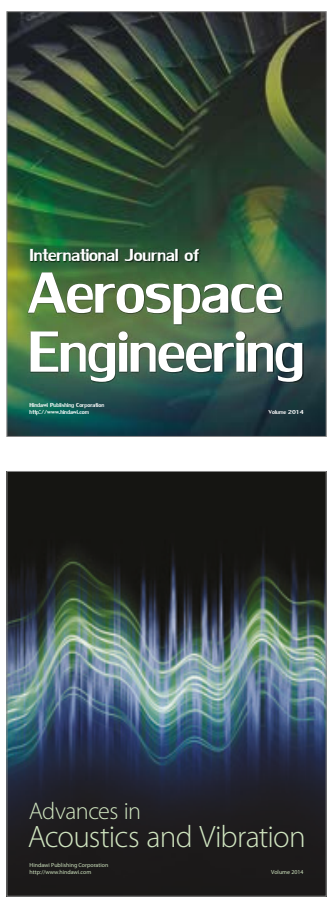

Sensor Networks 